\title{
ESTUDIO DE EFICACIA DE LA TÉCNICA DEL MAPA CONCEPTUAL Y EL CUESTIONARIO COMO MODELO DE ENSEÑANZA Y APRENDIZAJE SIGNIFICATIVO EN LOS ESTUDIANTES DE ÚLTIMO NIVEL DE UN GRUPO CONTROL Y EXPERIMENTAL DEL COLEGIO VÍCTOR FÉLIX GÓMEZ NOVA
}

\begin{abstract}
STUDY OF EFFECTIVENESS OF THE TECHNIQUE OF THE CONCEPTUAL MAP AND THE QUESTIONNAIRE AS A MODEL OF TEACHING AND SIGNIFICANT LEARNING IN THE LATEST LEVELS OF A CONTROL AND EXPERIMENTAL GROUP OF THE COLEGIO VÍCTOR FÉLIX GÓMEZ NOVA
\end{abstract}

\author{
Sandra M. Pedraza-Silva ${ }^{1}$
}

\section{RESUMEN}

En la enseñanza tradicional los docentes buscan que el alumno aprenda los conceptos en forma memorística, en la que consideran la forma más rápida de la instrucción, bajo el argumento del tiempo límite que se tiene asignado para cubrir el programa de estudio de la asignatura impartida. Partiendo de la necesidad de mejorar el almacenamiento de información a largo plazo, se desea aprovechar una herramienta que los docentes han empleado, y son los mapas conceptuales. La investigación fue realizada en el Colegio Víctor Félix Gómez Nova en el último año de escolaridad en la asignatura de Tecnología e Informática con estudiantes en edades comprendidas entre los 16 y 17 años, contó con un Grupo Experimental - GE de 30 estudiantes y un Grupo Control - GC de 17 estudiantes. Para indagar sobre las percepciones de los estudiantes frente a la técnica de mapas de conceptos se utilizó una muestra probabilística de 57 estudiantes.

PAlabras ClaVE: Mapa Conceptual, Metodología Cuantitativa, Escala Likert, Aprendizaje Significativo.

1 Universidad de Baja California - Tepic - Mexico. 


\begin{abstract}
In traditional teaching teachers seek that the student learn the concepts in a rote, in which they consider the fastest way of instruction, under the argument of the time limit that is allocated to cover the program of study of the subject taught. Starting from the need to improve the storage of information in the long term, we want to take advantage of a tool that teachers have used, and are conceptual maps. The research was conducted at the Víctor Félix Gómez Nova School in the last year of schooling in the subject of Technology and Computer Science with students between the ages of 16 and 17, it had an Experimental Group - GE of 30 students and a Control Group -GC of 17 students. To investigate the perceptions of the students in front of the technique of concept maps, a probabilistic sample of 57 students was used.
\end{abstract}

KEY WORDS: Conceptual Map, Quantitative Methodology, Likert Scale, Significant Learning.

\title{
INTRODUCCIÓN
}

La investigación fue realizada en el Colegio Víctor Félix Gómez Nova en el nivel medio, caracterizándose por ser una institución de carácter oficial, con modalidad académica ubicada en el municipio de Piedecuesta, Santander en Colombia. Dividida en dos sedes educativas y tres jornadas: por la mañana, por la tarde y por la noche el bachillerato. Cuenta con 19 aulas en la sede A y 17 en la sede B. La planta docente la componen en general 90 profesores de tiempo completo. El promedio de estudiantes por grupo es de 40 alumnos para una población global de 3,900 estudiantes de los cuales 300 son de la sección nocturna, los indicadores de índices de deserción escolar son bajos.

En la presente investigación se utilizó la metodología cuantitativa, con ayuda de cuestionarios como la escala Likert presentada por Cuervo (2009), donde se fijan indicadores de actitud hacia la construcción de mapas. Para validar las hipótesis de trabajo en las muestras control y experimental se realizó una prueba estadística que suele utilizarse en este tipo de diseño en la que se comparan los grupos experimental y control a partir de una prueba $t$ de Student para medias y para el coeficiente de correlación de Pearson. Al finalizar las secuencias didácticas en cada grupo se diseñó una prueba de conocimientos fundada en la construcción del mapa conceptual y otra en el diseño de un cuestionario. A partir del análisis de los resultados obtenidos en esta investigación se encontró una diferencia significativa en los tratamientos, determinando estadísticamente la efectividad de los mapas conceptuales como instrumento de almacenamiento de la información en la memoria a largo plazo. 
Para realizar el análisis de los efectos en la aplicación de dos estrategias de enseñanza en el rendimiento de estudiantes de bajo promedio escolar, se utilizó la metodología cuantitativa como modelo del proceso de búsqueda del conocimiento que se generó a partir del problema planteado, tomando como fuente de información: el examen de conocimientos aplicado al grupo experimental y de control con estudiantes de undécimo grado del Colegio Víctor Félix Gómez Nova, además de las respuestas a los cuestionarios con el diseño de la escala Likert presentado por Cuervo (2009), basados en indicadores de actitud hacia la construcción de mapas conceptuales por parte de los estudiantes

\section{Hipótesis}

Se planteó como hipótesis de trabajo, referente a si existen diferencias significativas al aplicar la estrategia de mapas conceptuales sobre la enseñanza y cómo afecta el rendimiento de los estudiantes con bajo promedio escolar. Para ello se definió un objetivo general de investigación y se determinó la efectividad de los mapas conceptuales como instrumento de almacenamiento de la información en la memoria a largo plazo; entre otros verificar como las herramientas tecnológicas, pueden facilitar y motivar en los estudiantes la construcción de mapas conceptuales.

\section{METODOLOGÍA}

La investigación pretende explicar cómo una estrategia de enseñanza puede ayudar a mejorar el rendimiento de los estudiantes con deficiencias conceptuales, por lo tanto se basa en el paradigma explicativo que conlleva la aplicación de información de corte cuantitativo para tratar de desarrollar el problema planteado. Este modelo denominado positivista, se ha impuesto como método científico en las ciencias naturales y más tarde en la educación, se caracteriza por la búsqueda de un conocimiento sistemático, comprobable, comparable, medible y replicable, lo cual nos lleva a emplear un tipo de enfoque cuantitativo, ya que se desean realizar mediciones y comparaciones de tipo experimental, buscando demostrar la existencia de diferencias significativas al aplicar la estrategia de mapas conceptuales sobre la enseñanza, y cómo se ve afectado el rendimiento de los estudiantes con bajo promedio escolar. En consideración, Pérez (2008) plantea que:

"Los mapas conceptuales no son una manera distinta de disponer, arreglar o acomodar los contenidos. representan una herramienta poderosa para desarrollar destrezas y capacidades cognitivas del pensamiento. Detrás de ellos está un cuerpo teórico riguroso que los 
sustenta. enseñar en el aula con una didáctica apoyada en mapas contribuye al aprendizaje de contenidos, pero la construcción de estas estructuras y su puesta en marcha en el aula requiere de una reflexión profunda por parte del docente".

La prueba estadística que suele utilizarse en este tipo de diseño en la que se comparan los grupos experimental y control es la prueba t de Student. Al respecto, Hernández, Fernández y Baptista (2014) la define como una prueba estadística para evaluar si dos grupos difieren de manera significativa respecto a sus medias basadas en una distribución muestral o poblacional.

Para el caso de la muestra representativa de la prueba Likert se tomaron 57 estudiantes al azar de una población de 115 estudiantes, estos cálculos partieron del análisis que se demuestra en el aparte de resultados.

En esta investigación se aplicó una prueba no estandarizada elaborada por los docentes de las diferentes sedes, colectiva porque serán muchos los examinados, no verbal o de ejecución al pedirle a los sustentantes señalar las respuestas correctas, de tipo cognitivo en la que se intenta cuantificar los procesos y productos de la actividad mental, y por último de rendimiento en la que se evalúa el conocimiento de algún tema.

El mismo autor caracteriza las pruebas afectivas, diseñadas para evaluar intereses, actitudes, valores, motivos y otras características de personalidad no cognoscitivas, motivo por el cual surge el siguiente apartado. La selección de la encuesta o escala Likert como herramienta para el estudio descriptivo de las actitudes de los estudiantes en la construcción de mapas conceptuales podría plantearse como un prediagnóstico o postdiagnóstico al finalizar el experimento o durante el mismo, sin embargo se optó por realizar la prueba durante la secuencia didáctica en los grupos. Las actitudes no son susceptibles de medición de forma directa, por ello se recurre a formas de medición indirecta, puesto que a partir del diseño de una escala de opinión se puede inferir sobre ellas. Al finalizar las secuencias didácticas en cada grupo se diseñó una prueba de conocimientos fundada en la construcción del mapa conceptual y otra en el diseño de un cuestionario.

\section{RESULTADOS}

En esta etapa se recolectaron los datos sobre las variables involucradas, en la que se elaboraron y aplicaron los instrumentos diseñados por las sedes: la escala Likert y el examen escrito. Con la finalidad de determinar el grado en que los reactivos de la escala Likert están correlacionados entre sí, se realizó un análisis de 
consistencia interna, obteniendo un valor de del coeficiente de alfa de Cronbach muy alto, $\alpha=0,94860$; lo cual indica confiabilidad entre los reactivos diseñados para conocer las actitudes de los estudiantes frente a la construcción de mapas conceptuales y las estrategias que han seguido sus maestros para implementarlas.

Se usó el coeficiente de correlación de Pearson y una prueba de consistencia interna mediante la prueba $t$ de Student, ambas técnicas demostraron la poca discriminación de algunos reactivos. Al respecto, Camacho (2007) define que:

"Un coeficiente de correlación se dice que es significativo si se puede afirmar, con una cierta probabilidad, que es diferente de cero. Más estrictamente, en términos estadísticos, preguntarse por la significación de un cierto coeficiente de correlación no es otra cosa que preguntarse por la probabilidad de que tal coeficiente proceda de una población cuyo valor sea de cero".

En consideración se definen dos hipótesis posibles, así:

$\mathrm{H}_{\mathrm{o}}: \mathrm{r}_{\mathrm{xy}}=0$, entonces el coeficiente de correlación obtenido procede de una población cuyo coeficiente de correlación es cero, $\rho=0$.

$\mathrm{H}_{1}: \mathrm{r}_{\mathrm{xy}} \neq 0$, entonces el coeficiente de correlación obtenido procede de una población cuyo coeficiente de correlación es distinto de cero, $\rho \neq 0$.

Desde el supuesto de la Hipótesis nula se demuestra que la distribución muestral de correlaciones procedentes de una población caracterizada por una correlación igual a cero, $\rho=0$, sigue una ley de Student con $n-2$ grados de libertad, de media el valor poblacional y desviación tipo:

$t_{\text {experimental }}>t_{n-2}$, entonces se rechaza la Hipótesis nula. La correlación obtenida no procede de una población cuyo valor $\rho_{x y}=0$. Por tanto las variables están relacionadas.

$t_{\text {experimental }} \leq t_{n-2}$, entonces se acepta la Hipótesis nula. La correlación obtenida procede de una población cuyo valor $\rho_{\mathrm{xy}}=0$. Por tanto ambas variables no están relacionadas.

$$
t_{\text {experimental }}=\frac{r_{x y} \cdot \sqrt{n-2}}{\sqrt{1-r_{x y}}}
$$

La población de análisis en la prueba tipo Likert fue del grado undécimo, de donde de una población total de 115 estudiantes, se tomó una muestra representativa de 57 estudiantes de manera probabilística, con un nivel de confianza del 95\%. En este caso desconocemos la varianza de la población, el cálculo se resume: 


$$
n=\frac{p \cdot q \cdot z^{2} \cdot N}{N \cdot e^{2}+z^{2} \cdot p \cdot q}=\frac{0,92 \cdot 0,08 \cdot 3,84 \cdot 115}{(115 \cdot 0,0025)+(3,84 \cdot 0,92 \cdot 0,08)} \sim 57
$$

Se hace una elección de un nivel de significación, del 0,05, respecto a un nivel de confianza del 95\% para una prueba de dos colas. Así el valor de $t_{55}=2,004$ que sirve de referente para la comparación de los valores experimentales del estadístico $t$ de Student para cada reactivo de la prueba Likert y de este modo decidir sobre su discriminación.

Frente a las 32 preguntas iniciales que indagaron sobre la actitud de los estudiantes hacia la construcción de mapas de conceptos, luego del análisis de discriminación se eliminaron solo 6 preguntas, al final la nueva prueba de percepciones para futuras investigaciones sobre el tema quedó en 26 reactivos.

Por otra parte se prepararon las mediciones obtenidas por medio de matrices de información en la que se codificaron los datos a fin de que se puedan analizar fácilmente.

El análisis de resultados de la postprueba, conclusiones y análisis de hipótesis en la sede luego de aplicar las estrategias y secuencias de aula:

Este análisis muestra los resultados de realizar una prueba de hipótesis relativa a la diferencia entre dos medias $\mu 1-\mu 2$ de muestras provenientes de distribuciones normales.

Las gráficas 1 y 2 prueban la distribución normal de los datos, junto con los estadísticos de los datos de los dos grupos de análisis.

El sesgo. En consideración, Lind, Marchal y Wathen (2012) definen que:

"Un conjunto de valores se encuentra sesgado a la derecha o positivamente sesgado si existe un solo pico y los valores se extienden mucho más allá a la derecha del pico que a la izquierda de éste. En este caso la media es más grande que la mediana. En una distribución negativamente sesgada existe un solo pico, pero las observaciones se extienden más a la izquierda, en la dirección negativa, que a la derecha".

En la Gráfica 1, se observa un sesgo positivo y en la Gráfica 2, se identifica un sesgo negativo ya que las observaciones se concentran más hacia la izquierda de la distribución. A partir de los datos anteriores, tenemos para el grupo control que $\bar{X} \geq M_{e}=M_{o}$, por lo tanto la distribución es asimétrica positiva; en el caso del grupo experimental se tiene $\quad \bar{X} \leq M_{e}=M_{o}$, por lo tanto la distribución es asimétrica negativa. 


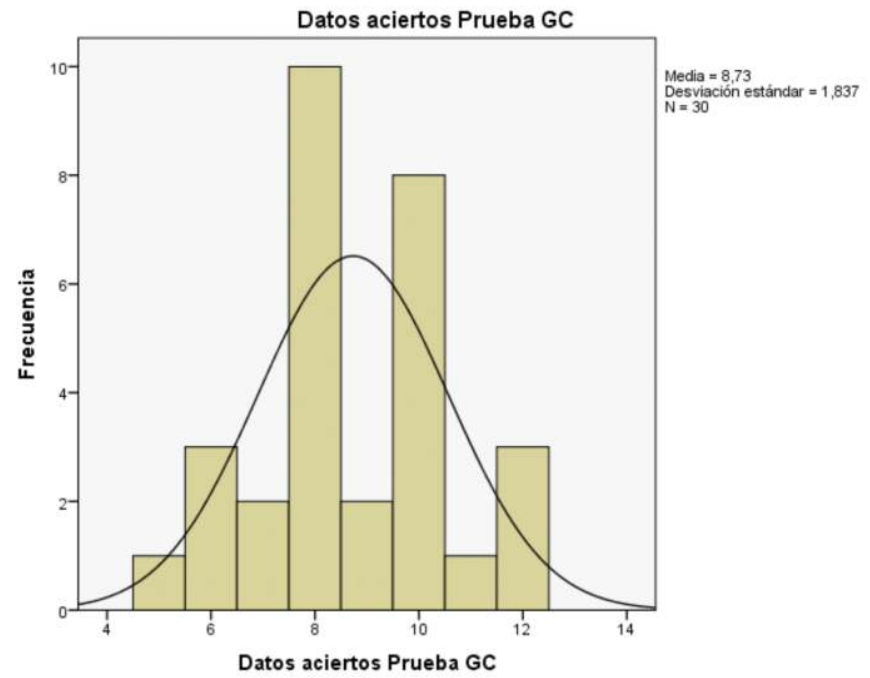

Gráfica 1. Distribución normal de los datos para el Grupo Control.

Fuente: Elaboración propia con base en: IBM (2014). IBM SPSS Statistics -ersión 23-,USA.

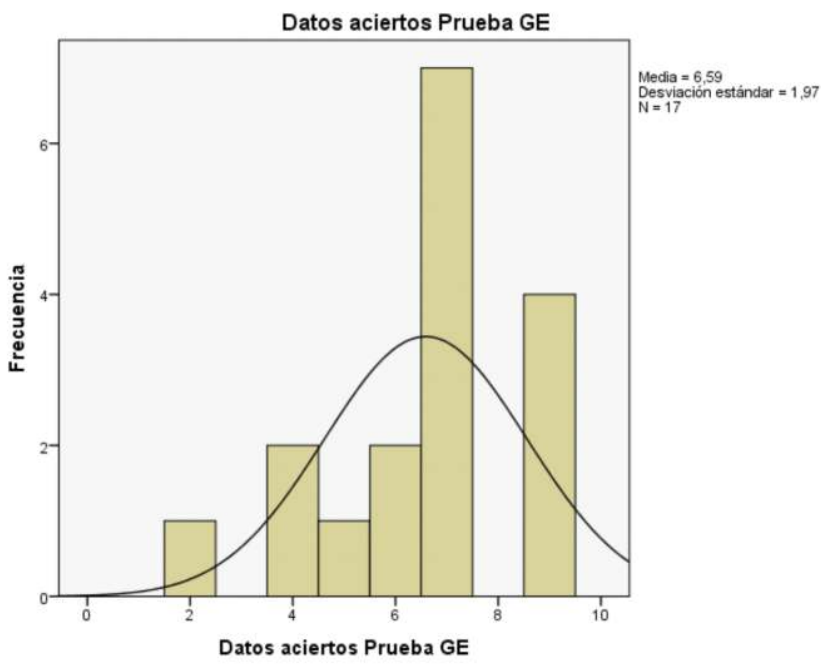

Gráfica 2. . Distribución normal de los datos para el Grupo Experimental. Fuente: Elaboración propia con base en: IBM (2014). IBM SPSS Statistics -ersión 23-,USA. 
Tabla 2

TABLA DE DESCRIPTIVOS ESTADÍSTICOS PARA EL GRUPO CONTROL Y EXPERIMENTAL

\begin{tabular}{|l|l|l|}
\hline & Grupo Control & Grupo experimental \\
\hline Recuento & 30 & 17 \\
\hline Promedio $-\bar{X}$ & 8,73333 & 6,58824 \\
\hline Mediana $-\mathrm{M}_{\mathrm{e}}$ & 8 & 7 \\
\hline Moda $-\mathrm{M}_{\mathrm{o}}$ & 8 & 7 \\
\hline Desviación Estándar & 1,83704 & 1,97037 \\
\hline Coeficiente de Variación & $21,0348 \%$ & $29,9074 \%$ \\
\hline Mínimo & 5,0 & 2,0 \\
\hline Máximo & 12,0 & 9,0 \\
\hline Rango & 7,0 & 7,0 \\
\hline Sesgo Estandarizado & 0,0647933 & $-1,23256$ \\
\hline Curtosis Estandarizada & $-0,519364$ & 0,354946 \\
\hline
\end{tabular}

Fuente: Elaboración propia con base en: StatGraphics (2009). StatGraphics Centurion XVI-Versión 16-, USA.

La curtosis. De acuerdo a la Tabla 2, para este análisis debemos considerarla, como el agrupamiento de los datos que se da alrededor del valor central. Al respecto, Spiegel y Stephens (2009) definen que la curtosis indica qué tan puntiaguda es una distribución; esto por lo regular es en relación con la distribución normal. Entre tanto, a una distribución que tiene un pico relativamente alto se le suele llamar leptocúrtica, si de otro modo, es relativamente aplastada se dice platicúrtica. Luego una distribución normal, que no es ni puntiaguda ni muy aplastada se le denomina mesocúrtica.

Para el cálculo de la curtosis se emplea el cuarto momento respecto de la media, esta medida corresponde a $g_{\text {control }}=-0,519364$ y $g_{\text {experimental }}=0,354946$.

De acuerdo al criterio de Gorgas, Cardiel y Zamorano (2011) se tiene que

"El coeficiente cuando tiene valores grandes, más puntiaguda es la distribución de los datos en su gráfica, si llega a tener exactamente un valor de 3, la distribución es del tipo mesocúrtica o normal, para el caso que sea mayor que 3 es del tipo leptocúrtica y si es inferior a 3 es del tipo platicúrtica".

Ya que, $g_{\text {control }}<3$, la distribución representada en la Gráfica 1, es del tipo platicúrtica, es decir la curva es aplastada. En el otro caso, $g_{\text {experimental }}>3$, la distribución representada en la Gráfica 2, es del tipo leptocúrtica, es decir la curva es picuda. 
Prueba de Hipótesis. Debemos probar que existen diferencias significativas al aplicar la estrategia de mapas conceptuales sobre la enseñanza y cómo afecta el rendimiento de los estudiantes con bajo promedio escolar.

Situación estadística: Se realiza una prueba de hipótesis - t de Student-, realizando la validación de una hipótesis de forma manual, apoyada con IBM SPSS y StatGraphics Centurion.

Haciendo el análisis de la información tenemos que:

$\mathrm{n}$ : es el tamaño de la muestra.

$z$ : es el nivel de confianza.

$\mu$ : media poblacional.

$\bar{x}$ : media muestral de los grupos del experimento.

$\sigma$ : es la desviación típica.

$s^{2}:$ es la varianza.

$\alpha$ : nivel de significación.

$t_{n_{1}+n_{2}-2}$ : t de Student con $n_{1}+n_{2}-2$ grados de libertad.

Para el experimento propuesto tenemos que las respuestas a los reactivos del grupo control se distribuyen normalmente y son:

$$
\begin{aligned}
\bar{\mu}_{1} & =8,7 \\
s_{1} & =1,84 \\
n_{1} & =30
\end{aligned}
$$

Para el experimento propuesto tenemos que las respuestas a los reactivos del grupo experimental se distribuyen normalmente y son:

$$
\begin{aligned}
& \bar{\mu}_{2}=6,6 \\
& s_{2}=1,97 \\
& n_{2}=17
\end{aligned}
$$

Al respecto del contraste de hipótesis, Martínez (2012) define que las pruebas de hipótesis, denominadas también pruebas de significación tienen como objeto principal evaluar suposiciones o afirmaciones acerca de los valores estadísticos de la población, denominados parámetros. En este sentido, Hernández, Fernández y Baptista (2014) afirman que: 
"Las hipótesis, en el enfoque cuantitativo, se someten a prueba en la realidad cuando se implementa un diseño de investigación, se recolectan datos con uno o varios instrumentos de medición, y se analizan e interpretan esos mismos datos".

Para contrastar las muestras se siguió el siguiente procedimiento para el cálculo del estadístico-t-:

1. Se formula una hipótesis nula y una hipótesis alternativa.

2. Se elige una distribución de muestreo apropiada, en el caso de estudio $\mathrm{n}<120$, por tanto es recomendable una distribución $\mathrm{t}$ de Student.

3. Elección de un nivel de significación, como en el ejemplo de la investigación es del 0,05-95\% de confianza- para una prueba de una cola.

4. Se desarrollan cálculos de la estadística de la prueba, este se realiza con la fórmula de - $\mathrm{t}$ - en la cual se introducen los valores encontrados en el estudio, como sigue:

Las dos hipótesis a ser evaluadas aquí son:

Hipótesis nula $-\mathrm{Ho}-: \mu 1-\mu 2=0$

Media muestral de aciertos del Grupo Control: $\mu 1$

Hipótesis alterna $-\mathrm{Ha}-: \mu 1-\mu 2 \neq 0$

Media muestral de aciertos del Grupo Experimental: $\mu 2$

Dada una muestra -Grupo Control- de 30 reactivos con una media de aciertos de respuestas correctas de 8,7 y una desviación estándar de 1,84 y una segunda muestra -Grupo Experimental-de 17 observaciones con una media de 6,6 y una desviación estándar de 1,97; el procedimiento para el cálculo del estadístico $-\mathrm{t}-\operatorname{con} \mathrm{n}_{1}+\mathrm{n}_{2}-2$ grados de libertad, es:

$$
t_{n_{1}+n_{2}-2}=\frac{\mu_{1}-\mu_{2}}{\sigma \mu_{1}-\mu_{2}}
$$

En la expresión, el numerador representa la media aritmética de las diferencias entre los momentos de los grupos de comparación del experimento, el denominador es el error estándar de sus diferencias que se puede calcular con la ecuación:

$$
\sigma \mu_{1}-\mu_{2}=\sqrt{\frac{n_{1} s_{1}^{2}+n_{2} s_{2}^{2}}{n_{1}+n_{2}-2}} \cdot \sqrt{\frac{n_{1}+n_{2}}{n_{1} n_{2}}}
$$


Las -n-son los tamaños de las muestras del grupo experimental y control y las -s- representan las desviaciones estándar respectivamente.

$$
\text { Así, } \quad t_{45}=\frac{\mu_{1}-\mu_{2}}{\sqrt{\frac{n_{1} s_{1}^{2}+n_{2} s_{2}^{2}}{n_{1}+n_{2}-2}} \cdot \sqrt{\frac{n_{1}+n_{2}}{n_{1} n_{2}}}}=\frac{2,1}{\sqrt{\frac{167,5433}{45}} * \sqrt{\frac{47}{510}}}=3,585074443
$$

Al reemplazar datos en las fórmulas, obtenemos un valor para el estadístico -t, que luego se compara con el valor dado en la tabla de distribución de valores student, tomando el nivel de significación elegido $-0,05-$ y considerando los grados de libertad que se definen con la expresión gl $=\mathrm{n}_{1}+\mathrm{n}_{2}-2=30+17-2=$ 45. Calculando este estadístico de forma experimental se obtiene,

$$
t_{\text {experimental }}=3,585074443
$$

En la expresión, el numerador representa la media aritmética de las diferencias entre los momentos de los grupos de comparación, el denominador es el error estándar de las medias muestrales. Donde -n- es el tamaño de las muestra respectiva y $-\sigma-$ representa la desviación estándar. Al respecto, Mendenhall, Beaver y Beaver (2010) sostienen que para efectuar la prueba t para estas dos muestras independientes, se debe suponer que las poblaciones muestreadas son normales y tienen la misma varianza o son casi iguales.

Luego al ubicar en la Tabla 3, los grados de libertad y el nivel de significación de 0,05 y el cruce indica un valor para $t=1,679$ para la prueba de una cola. Las hipótesis que se han definido son:

$\mathrm{H}_{\mathrm{o}}$ : Si no se presentan diferencias significativas al aplicar la estrategia de mapas conceptuales sobre la enseñanza, no se favorece el rendimiento de los estudiantes con bajo promedio escolar.

$\mathrm{H}_{\mathrm{A}}$ : Si se presentan diferencias significativas al aplicar la estrategia de mapas conceptuales sobre la enseñanza, se favorece el rendimiento de los estudiantes con bajo promedio escolar.

Si asumimos un nivel de significación $\alpha=0.05$, en la tabla de distribución de probabilidad de $t$ de Student, observamos que el valor de $-t-$ para $\alpha=0.05$ y 45 grados de libertad es, $t_{\text {tabla }}=1.679$. Como, $t_{\text {experimental }}>t_{\text {tabla }}$, tenemos que rechazar la Hipótesis Nula y aceptar la Hipótesis Alternativa dentro de los términos probabilísticos seleccionados, en tal caso como se presentan diferencias significativas al aplicar la estrategia de mapas conceptuales sobre la enseñanza, se favorece el rendimiento de los estudiantes con bajo promedio escolar. 
Tabla 3

DISTRIBUCIÓN T DE STUDENT

\begin{tabular}{|c|c|c|c|c|c|c|}
\hline & & & $\begin{array}{ll}0 & t \\
c \text { cola derecha }\end{array}$ & Prvebe of & & \\
\hline \multirow{4}{*}{ gl } & $80 \%$ & $90 \%$ & $95 \%$ & $98 \%$ & $99 \%$ & $99.9 \%$ \\
\hline & \multicolumn{6}{|c|}{ Nivel de significancia de una prueba de una cola,? } \\
\hline & 0.10 & 0.05 & 0.025 & 0.01 & 0.005 & 0.005 \\
\hline & \multicolumn{6}{|c|}{ Nivel de significancia de una prueba de dos colas,? } \\
\hline & 0.20 & 0.10 & 0.05 & 0.02 & 0.01 & 0.001 \\
\hline 36 & 1.306 & 1.638 & 2.028 & 2.434 & 2.719 & 3.582 \\
\hline 37 & 1.305 & 1.637 & 2.026 & 2.431 & 2.715 & 3.574 \\
\hline 38 & 1.304 & 1.636 & 2.024 & 2.429 & 2.712 & 3.566 \\
\hline 39 & 1.304 & 1.635 & 2.023 & 2.426 & 2.708 & 3.558 \\
\hline 40 & 1.303 & 1.634 & 2.021 & 2.423 & 2.704 & 3.551 \\
\hline 41 & 1.303 & 1.693 & 2.020 & 2.421 & 2.701 & 3.544 \\
\hline 42 & 1.302 & 1.632 & 2.018 & 2.418 & 2.698 & 3.538 \\
\hline 43 & 1.302 & 1.681 & 2.017 & 2.416 & 2.695 & 3.532 \\
\hline 44 & 1.301 & 1.630 & 2.015 & 2.414 & 2.692 & 3.526 \\
\hline 45 & 1.301 & 1.679 & 2.014 & 2.412 & 2.690 & 3.520 \\
\hline 46 & 1.300 & 1.679 & 2.013 & 2.410 & 2.687 & 3.515 \\
\hline 47 & 1.300 & 1.678 & 2.012 & 2.403 & 2.685 & 3.510 \\
\hline 48 & 1.299 & 1.677 & 2.011 & 2.407 & 2.682 & 3.505 \\
\hline 49 & 1.299 & 1.677 & 2.010 & 2.405 & 2.680 & 3.500 \\
\hline 50 & 1.299 & 1.676 & 2.009 & 2.403 & 2.678 & 3.496 \\
\hline 51 & 1.298 & 1.675 & 2.008 & 2.402 & 2.676 & 3.492 \\
\hline 52 & 1.298 & 1.675 & 2.007 & 2.403 & 2.674 & 3.488 \\
\hline 53 & 1.298 & 1.674 & 2.006 & 2.399 & 2.672 & 3.484 \\
\hline 54 & 1.297 & 1.674 & 2.005 & 2.397 & 2.670 & 3.480 \\
\hline 55 & 1.297 & 1.673 & 2.004 & 2.396 & 2.668 & 3.476 \\
\hline 56 & 1.297 & 1.673 & 2.003 & 2.395 & 2.667 & 3.473 \\
\hline 57 & 1.297 & 1.672 & 2.002 & 2.394 & 2.665 & 3.470 \\
\hline 58 & 1.296 & 1.672 & 2.002 & 2.392 & 2.663 & 3.466 \\
\hline 59 & 1.296 & 1.671 & 2.001 & 2.391 & 2.662 & 3.463 \\
\hline 60 & 1.296 & 1.671 & 2.000 & 2.393 & 2.660 & 3.460 \\
\hline 61 & 1.296 & 1.670 & 2.000 & 2.389 & 2.659 & 3.457 \\
\hline 62 & 1.295 & 1.670 & 1.999 & 2.383 & 2.667 & 3.454 \\
\hline 63 & 1.296 & 1.669 & 1998 & 2.387 & 2.656 & 3.452 \\
\hline 64 & 1.296 & 1.669 & 1998 & 2.385 & 2.655 & 3.449 \\
\hline 65 & 1.295 & 1.669 & 1.997 & 2.385 & 2.654 & 3.447 \\
\hline
\end{tabular}

Fuente: Elaboración propia con base en: Lind, Marchal y Wathen (2012). Estadística aplicada a los negocios y la economía, México. 
Este análisis es corroborado por el contraste de muestras del análisis estadístico usando IBM SPSS Statistics, se muestra en la Tabla 4.

Tabla 4

ANÁLISIS DE PRUEBA DE MUESTRA INDEPENDIENTE PARA ACIERTOS POR ESTUDIANTES GRUPO CONTROL Y EXPERIMENTAL

\begin{tabular}{|c|c|c|c|}
\hline \multirow[b]{2}{*}{ Datos aciertos Prueba GC y GE } & \multicolumn{3}{|c|}{ Prueba t para la igualdad de medias } \\
\hline & gl & $\begin{array}{r}\text { Sig. } \\
\text { (bilateral) }\end{array}$ & Diferencia de medias \\
\hline Se asumen varianzas iguales & 45 & 0,001 & 2,1451 \\
\hline No se asumen varianzas iguales & 31,436 & 0,001 & 2,1451 \\
\hline Grupo Control y Experimental & $\mathrm{N}$ & Media & Desviación estándar \\
\hline GC & 30 & 8,7333 & 1,83704 \\
\hline GE & 17 & 6,5882 & 1,97037 \\
\hline
\end{tabular}

Fuente: Elaboración propia con base en: IBM (2014). IBM SPSS Statistics -Versión 23-, USA.

El valor de significancia asintótica bilateral o $\mathrm{p}$-valor $=0,001$, comparando con el nivel de significancia $-2 \alpha$ - para una cola en la distribución t de Student, que corresponde al $10 \%=0,1$ concluimos que $\mathrm{p}$-valor $<2 \alpha=0,1$ con lo cual debemos aceptar la Hipótesis Alternativa $-\mathrm{H}_{\mathrm{A}}$, dentro de los términos probabilísticos seleccionados.

Estos resultados fueron contrastados con un análisis de dos muestras desarrollado en el software StatGraph Centurion para la sede de la investigación, utilizando tablas de estadísticos y de frecuencias, además de histogramas con polígonos de frecuencia. Una extensa información se presenta en el apéndice $G$ de este documento para la sede de estudio, utilizando tablas de estadísticos y de frecuencias, además de histogramas con polígonos de frecuencia.

\section{DISCUSIÓN}

A partir del análisis de los resultados obtenidos en esta investigación se encontró una diferencia significativa en los tratamientos en la sede CVFG determinando estadísticamente la efectividad de los mapas conceptuales como instru- 
mento de almacenamiento de la información en la memoria a largo plazo. Coincidiendo con Chiou (2008), quien menciona que la adopción de una estrategia de mapas conceptuales pueden mejorar significativamente el rendimiento académico de aprendizaje en comparación al uso de un método de enseñanza tradicional expositivo.

Los estadísticos de prueba nos señalan la existencia de un aprendizaje significativo en los alumnos que se sometieron a la elaboración de un mapa conceptual con respecto a los que elaboraron cuestionarios al obtener en las tres sedes una mayor frecuencia de estudiantes con mayores calificaciones, producto de la rigurosa secuencia didáctica que se llevó a cabo en los tres planteles educativos. Al respecto, Pozo (1994) hace referencia a la teoría de Ausubel en cuanto a la formación del aprendizaje significativo, en la que se precisa de una instrucción formalmente establecida, que presente de modo organizado y explicito la información que debe de equilibrar las estructuras existentes.

En cuanto a los indicadores encontrados sobre la actitud de construir mapas conceptuales en los alumnos del plantel educativo sujetos a la investigación se encontró que existe un conocimiento bastante aceptable sobre esta estrategia de aprendizaje, aunque su uso no es muy común por parte de las estudiantes.

Por lo anterior se recomienda realizar una serie de investigaciones en la que se relacionen secuencias didácticas con procesos de metacognición o de aprendizaje autorregulado que ayuden a concientizar a los alumnos de bajo rendimiento académico sobre sus procesos cognitivos al realizar estas tareas de aprendizaje.

Tanto el grupo control como experimental fueron grupos inicialmente seleccionados con base a promedios bajos; mediante las secuencias didácticas se cumplió con el segundo objetivo que pretendía medir las diferencias entre el aprendizaje memorístico y el significativo, para ello se construyeron 2 tipos de postpruebas diferentes de acuerdo al grupo seleccionado y la estrategia didáctica a desarrollar.

En el caso de esta sede se recolectó la información mediante la sistematización de los dos cuestionarios en plataforma Survey, luego se procedió a generar reportes y a realizar el análisis respectivo en tablas de frecuencia, fue más confiable realizarlo de esta forma se ahorró mucho tiempo en el análisis de resultados.

Se cumplió desde luego con el primer objetivo que fue determinar la efectividad de los mapas conceptuales sobre el aprendizaje y fue ampliada con la inda- 
gación en la escala de categorías Likert que se implementó de forma electrónica y donde se evidenció un promedio de 198,982456140351 puntos acumulados en la prueba de 280 puntos posibles -56 reactivos con un valor de 5 puntos como máximo-; lo cual es satisfactorio a nivel general o permite ver la aceptación de la técnica del mapa conceptual.

Para el segundo objetivo se cumplió con lo propuesto y fue indagar sobre que tanto habían aprendido los estudiantes luego de realizadas las secuencias didácticas; se lograron competencias de tipo tecnológico, se mejoró la lectura a través del libro de los Seis sombreros para pensar y se usaron por primera vez en el aula pruebas electrónicas confiables.

Para el tercer objetivo se diseñó una secuencia didáctica en cada caso que permitió llevar a cabo un proceso de casi un mes donde los estudiantes del grupo experimental aprendieron a construir mapas conceptuales y apropiarse de conceptos, razón de ello, es que se plantearon dos esquemas para llevar acabo los experimentos el uno uso las técnicas del cuestionario - GC-y el otro consideró la técnica de mapas conceptuales con ayuda de recursos como Cmaptools-GE-.

Para el cuarto objetivo se diseñaron indicadores de actitud hacia la construcción de mapas conceptuales, razón de ello, fue la prueba Likert desarrollada en una población de 57 estudiantes de los grados undécimos; en ella se concluyó que la mayoría de reactivos eran adecuados para desarrollar el test, se obtuvo en el cálculo del coeficiente de Pearson que 23 reactivos eran de confiabilidad media, 3 de confiabilidad aceptable, 20 de confiabilidad débil y muy pocos -10 reactivos-se debieron eliminar por su escasa confiabilidad.

En total se evaluaron 56 reactivos haciendo con esto que la prueba fuera lo más confiable posible; al realizar el análisis de confiabilidad de la Prueba obtuvimos un coeficiente de igual a 0,94866682942452, lo cual nos arroja un resultado muy alto entre 0,81 y 1 ; lo que indica que fue muy confiable realizar la Prueba con los reactivos propuestos luego de la eliminación de los menos confiables.

Para el quinto objetivo se implementó aplicaciones en software como CmapTools para la construcción de mapas conceptuales, cada estudiante del grupo experimental estuvo al frente de la construcción de varias versiones de mapas desarrollados con este ambiente, muchos de ellos lograron la construcción de mapas nivel 3, 4 y 5; se utilizó para ello el modelo topológico estructural que valora la complejidad de la construcción del mapa conceptual en 7 niveles-0 a 6-. 
En el nivel tres se evidenció que los estudiantes no relacionan las ramas de sus mapas conceptuales y son demasiado jerárquicos verticalmente. Para el nivel cuatro se evidenció uso del color, formas, conectores pero se carece de conexiones entre ramas del mapa.

Se presentaron algunos buenos mapas conceptuales entre algunos estudiantes, tenían buena organización, conexiones entre ramas, colores vistosos, profundidad del tema leído del libro de los seis sombreros para pensar de De Bono (1998). El contraste de hipótesis de las sedes se puede ver en el apéndice $H$.

Recomendable es que en el aula se manifiesten este tipo de estrategias, en las cuales se plantean secuencias didácticas, se formulan temas nuevos, se usan herramientas tecnológicas innovadoras y sobre todo se impulsa la creación de materiales, recursos y medios educativos que permiten centrar el interés de los estudiantes, permiten dar una evaluación pertinente de sus procesos con una consideración más clara de sus dificultades y fortalezas.

El diseño global del experimento dio fundamento a la realización de pruebas estadísticas fundadas en los principios de la investigación de Hernández y otros (2014); quienes brindan algunas pautas para el desarrollo de la investigación y uso de herramientas tecnológicas como StatGraph Centurion, ambos recursos sirvieron para potenciar y dar valor agregado a los análisis y pruebas de las hipótesis de los grupos control y experimental.

En dichos resultados constatamos la diferencia de las dos muestras al aceptarse la diferencia significativa entre las medias de los grupos frente a las respuestas correctas de los test, en consideración de la comparación de muestras el GC tiene una media de 8,7 y el GE una media de 6,5; el tamaño varía mucho, el grupo control es casi el doble del experimental -30 estudiantes GCy 17 en el GE-.

Estos promedios altos en el grupo control estarían sujetos a una observación que encontramos con algunos estudiantes que usan la técnica del mapa conceptual por sugerencia de otros estudiantes en cursos anteriores como estilo de estudio en algunas asignaturas, otra variable es que los estudiantes aceptados con promedios bajos por sus notas no necesariamente son los más malos, encontramos un caso de una estudiante que no le gustaba la metodología del docente en una de sus materias y razón de ello reprobó la asignatura y bajó su promedio académico, sin embargo es una estudiante que le gusta leer, es crítica y este año al cambiar de instructor de tecnología es muy sobresaliente; por lo tanto definir una 
muestra homogénea es una tarea bastante complicada que es determinada por más variables que las que se suponen inicialmente.

\section{CONCLUSIONES}

En cuanto a los indicadores encontrados sobre la actitud de construir mapas conceptuales en los estudiantes sujetos a la investigación se encontró que existe un conocimiento bastante aceptable sobre esta estrategia de aprendizaje, aunque su uso no es muy común por parte de las estudiantes. Por lo anterior se recomienda realizar una serie de investigaciones en la que se relacionen secuencias didácticas con procesos de metacognición o de aprendizaje autorregulado que ayuden a concientizar a los estudiantes de bajo rendimiento académico sobre sus procesos cognitivos al realizar estas tareas de aprendizaje.

Los docentes son responsables de impulsar este tipo de trabajo con las estudiantes, ellos deben cambiar la metodología de colocar un texto para que las estudiantes, construyan un mapa conceptual, le deben dar mayor importancia, al diseño, análisis, síntesis y revisión para que la estudiante le dé la importancia de revisarlo hasta que el resultado final sea el más adecuado. Esta apropiación que asuma el profesor y el gusto que el demuestre impregnará en las estudiantes, la idea de trabajar con esta metodología.

Aunque no es muy común el trabajo de mapas conceptuales por parte de las estudiantes, se puede observar que obtuvieron mejor rendimiento en la postprueba que las estudiantes del grupo de control, tales resultados, se deben tener en cuenta para comenzar a implementar este tipo de trabajo en las aulas de clase.

Los estadísticos de prueba nos señalan la existencia de un aprendizaje significativo en los alumnos que se sometieron a la elaboración de un mapa conceptual con respecto a los que elaboraron cuestionarios al obtenerse una mayor frecuencia de estudiantes con mayores calificaciones, producto de la rigurosa secuencia didáctica que se llevó a cabo en el plantel educativo. Al respecto, Pozo (1994) hace referencia a la teoría de Ausubel en cuanto a la formación del aprendizaje significativo, en la que se precisa de una instrucción formalmente establecida, que presente de modo organizado y explicito la información que debe de equilibrar las estructuras existentes.

Frente a la posibilidad de rechazar o no la hipótesis nula o considerar que esta es adecuada, debe establecerse una buena definición de las hipótesis y de los 
modelos estadísticos a considerar, sin embargo debe definirse en términos sencillos y sin tecnicismos las definiciones de las hipótesis para que estas puedan ser entendidas por otras personas, de este modo se establece un verdadero significado para la conclusión cuando se contrastan muestras. Cuando se quiere sustentar alguna aseveración, debe expresarse esta de tal modo que se convierta en la hipótesis alternativa, y posteriormente, luego de un análisis estadístico, esperarse que la hipótesis nula sea rechazada.

Para enfrentar el problema de comparar medias a través de la prueba t de Students con el software IBM SPSS Statistics debe necesariamente tenerse claridad en los datos recolectados, para que este pueda establecer los estadísticos necesarios para poder aceptar o no las hipótesis de estudio. En el caso de dos muestras, para realizar el contraste es más sencillo y posee los parámetros adecuados para establecer las pruebas de hipótesis. No obstante la pericia del investigador lleva a la formulación de un conjunto de datos que se comportan de manera adecuada a las condiciones del problema inicial, lo cual establece un buen punto de partida para simular las condiciones del problema inicial y comprobar la hipótesis nula.

Las tablas de las muestras, corroboran los estadísticos de resumen para cada categoría en la sede CVFGN. Incluye medidas de tendencia central, medidas de variabilidad y medidas de forma. De particular interés aquí son el sesgo estandarizado y la curtosis estandarizada, las cuales pueden utilizarse para determinar si la muestra proviene de una distribución normal. Valores de estos estadísticos fuera del rango de -2 a +2 indican desviaciones significativas de la normalidad, lo que tendería a invalidar cualquier prueba estadística con referencia a la desviación estándar. En este caso, el valor del sesgo estandarizado se encuentra dentro del rango esperado para datos provenientes una distribución normal. El valor de curtosis estandarizada se encuentra dentro del rango esperado para datos provenientes de una distribución normal por lo tanto los datos se comportan de manera normal.

En el caso del software StatGraphics Centurion, si se sospecha que los datos de estudio contienen valores atípicos inevitables, se puede ejecutar una prueba no paramétrica para comparar las medianas en lugar de las medias. Las pruebas no paramétricos no asumen que los datos provengan de una distribución normal y tienden a ser menos afectados por la presencia de valores atípicos, sin embargo es aconsejable cuando se tiene certeza de datos que podrían afectar sustancialmente las conclusiones finales. 
Es posible que cuando se realiza un análisis de datos de un experimento, se requieran en algunos casos ciertos subconjuntos de datos, el software estadístico IBM SPSS Statistics permite filtrar temporalmente un rango de datos. Para este propósito se utiliza la opción -Seleccionar casos- del menú -Datos-. En la ventana pertinente figuran cinco tipos de filtros, el de uso más común es el que permite personalización accesible desde la ventana de -Personalizar- en la opción -Si se satisface la condición-. A partir de las expresiones que se requieran y las condiciones se puede generar un filtro que incluye una o varias expresiones condicionales, las cuales pueden construirse a partir de variables ya definidas, junto con operadores lógicos y funciones predefinidas del software estadístico.

\section{REFERENCIAS BIBLIOGRÁFICAS}

Acosta, S. y Acosta, R. (2010). Los mapas conceptuales y su efecto en el aprendizaje biológico. Venezuela: Universidad del Zulia. Recuperado en: http://www.redalyc.org/pdf/737/73715084012.pdf (Mayo 25 de 2018)

Aiken, L. (2003). Tests psicológicos y evaluación (11va. Ed.) México: Pearson Educación. Recuperado en: http://www38.zippyshare.com/v/45791785/file.html (Mayo 28 de 2018)

Camacho, D. (2007). Coeficiente de correlación lineal de Pearson. Universidad de Sevilla. Recuperado en: https://personal.us.es/vararey/adatos2/correlacion.pdf (Mayo 13 de 2018)

Cañas, A. y Novak, J. (2006). Confiabilidad de una taxonomía topológica para mapas conceptuales. Florida Institute for Human and Machine Cognition -IHMC-, USA Norma L. San José, Costa Rica. Recuperado en: http://cmc.ihmc.us/cmc2006Papers/cmc2006p233.pdf (Mayo 24 de 2018)

Chiou, Ch. (2008). The effect of concept mapping on students' learning achievements and interests .Innovations in Education and Teaching International. Department of Accounting, National Changhua University of Education, Changhua, Taiwan. Recuperado en: http://citeseerx.ist.psu.edu/viewdoc/download?doi=10.1.1.467.4729\&rep=rep1\&type $=p$ df (Mayo 26 de 2018)

Cravalho, P. (2010). Learning statistics using concept maps: effects on anxiety and performance -Tesis de Maestría-. Departament of Psychology, San José State University. Recuperado en: http://scholarworks.sjsu.edu/cgi/viewcontent.cgi?article $=4802 \&$ context $=$ etd_theses (Mayo 20 de 2018)

De Bono, E. (1998). Seis sombreros para pensar. Barcelona: Granica Ediciones. Recuperado en: http://jorgeserrano.es/wp-content/uploads/2016/02/Edward-de-Bono-6-sombreros-parapensar.pdf (Mayo 22 de 2018)

Díaz, F. y Hernández, G. (2002). Estrategias docentes para un aprendizaje significativo: una interpretación constructivista (2da. ed.). Distrito Federal, México: Mc Graw Hill. Recuperado en: https://jeffreydiaz.files.wordpress.com/2008/08/estrategias-docentes-paraun-aprendizaje-significativo.pdf (Mayo 26 de 2018) 
Garza, R. y Leventhal, S. (1998). Aprender cómo aprender (3ra. ed.). Distrito Federal, México: Trillas.

Giroux, S. y Tremblay, G. (2004). Metodología de las ciencias humanas (1ra. Ed.). México: Fondo de Cultura Económico. Recuperado en: https:/es.scribd.com/doc/240021562/Metodologiade-Las-Ciencias-Humanas (Mayo 20 de 2018)

Gönen, S. (2006). Effects of concept maps, semantic networks and computer simulations on students' understandings of quantum physics. Journal of Science Education. ProQuest Education Journals.

Gorgas, J., Cardiel, N., y Zamorano, J. (2011). Estadística básica para estudiantes de ciencias. España: Universidad Complutense de Madrid. Recuperado en: http://pendientedemigracion.ucm.es/info/Astrof/users/jaz/ESTADISTICA/libro_GCZ200 9.pdf (Mayo 1 de 2018)

Hernández, R., Fernández, C. y Baptista, P. (2014). Metodología de la investigación (6ta. Ed.). México: McGRAW-HILL. Recuperado en: https://rive.google.com/file/d/OBx2rrMyYItoWVp1OEQ5ZldKNHc/view?usp=sharing (Mayo 13 de 2018)

IBM (2014). Guía breve de IBM SPSS Statistics 23. Recuperado en: $\mathrm{ftp} / / /$ public.dhe.ibm.com/software/analytics/spss/documentation/statistics/23.0/es/client/M anuals/IBM_SPSS_Statistics_Brief_Guide.pdf(Mayo 8 de 2018)

IBM (2014). IBM SPSS Statistics (Versión 23), USA [Software]. Recuperado en: http://www01.ibm.com/support/docview.wss?uid=swg24038592 (Mayo 9 de 2018)

IHMC CmapTools (2017). Software de mapeo de conceptos (Ver. 6.02). Florida: Institute for Human and Machine Cognition. Recuperado en: https://cmap.ihmc.us/cmaptools/cmaptools-download/ (Mayo 27 de 2018)

Lind, D., Marchal., W. y Wathen, S. (2012). Estadística aplicada a los negocios y la economía (15ava. Ed.). México: MacGrawHill. Recuperado en:

https://jaimesotou.files.wordpress.com/2011/05/libro-estadistica-aplicada-negocios.pdf (Mayo 22 de 2018)

Martínez, C. (2011). Estadística Básica Aplicada (4ta. ed.). Bogotá: Eco Ediciones. Recuperado en: https://xlibros.com/wp-content/uploads/2014/04/Estad\%C3\%ADstica-B\%C3\%A1sicaAplicada-FL_redacted.pdf (Mayo 24 de 2018)

Martínez, C. (2012). Estadística y muestreo (13va. ed.). Bogotá: Eco Ediciones. Recuperado en: https://wwwyyy.files.wordpress.com/2016/06/estadc3adstica-y-muestreo-de-ciromartc3adnez-b.pdf (Mayo 24 de 2018)

Mendenhall, W., Beaver, R. y Beaver, B (2010). Introducción a la probabilidad y la estadística (13va. Ed.). México: Cengage Learning Editores. Recuperado en:

http://investigadores.cide.edu/aparicio/data/refs/Mendenhall_Prob_Estadistica_13.pdf (Mayo 23 de 2018)

Ormrod, J. (2005). Aprendizaje humano (4ta. ed.). Madrid, España: Pearson Prentice Hall. Recuperado en: https://saberespsi.files.wordpress.com/2016/09/ellis-aprendizajehumano.pdf (Mayo 20 de 2018) 
Pérez, R. (2008). Mapas conceptuales como una manera de repensar la enseñanza. Gestión y Estrategia. Recuperado en: https:/es.scribd.com/document/180197242/MAPAS-EN-LAENSENANZA-MATE\# (Mayo 18 de 2018)

Pozo, J. (1994). Teorías cognitivas del aprendizaje (3ra. ed.). Madrid, España: Morata. Recuperado en: https://es.scribd.com/doc/73088854/POZO-J-I-2006-Teorias-Cognitivas-DelAprendizaje-Editorial-MORATA-Espana (Mayo 19 de 2018)

Spiegel, M. y Stephens, L. (2009). Estadística (4ta. Ed.). McGraw-Hill / Interamericana de México. Recuperado en: http://www10.zippyshare.com/v/89258660/file.html (Mayo 20 de 2018)

StatGraphics (2009). Manual del usuario. StatGraphics Centurion XVI -Versión 16-, USA. Recuperado en: http:/www.statgraphics.net/wp-content/uploads/2015/03/Centurion-XVIManual-Principal.pdf (Mayo 23 de 2018)

StatGraphics (2009). StatGraphics Centurion XVI -Versión 16-, USA [Software]. Recuperado en: http://www.statgraphics.net/descargas/ (Mayo 23 de 2018)

Anderson, D., Sweeny, D. y Williams, T. (2008). Estadística para administración y economía (10a. Ed.). México: CengageLearning Editores, S.A. Recuperado en: https://www.upg.mx/wpcontent/uploads/2015/10/LIBRO-13-Estadistica-para-administracion-y-economia.pdf (Junio 6 de 2018)

Martínez, C. (2012). Estadística y muestreo (13ava. Ed.). Bogotá: Ecoe Ediciones. Recuperado en: http://documents.mx/documents/estadistica-y-muestreo-13va-edicion-ciro-martinezbencardino-freelibrosorg.html (Junio 2 de 2018)

Moreno, E. (2008). Manual de uso de SPSS (1ra. Ed.).Madrid: Universidad Nacional de Educación a Distancia. Recuperado en: http://e-spacio.uned.es/fez/eserv/bibliuned:500727/Guia_ SPSS.pdf (Junio 1 de 2018)

Ochoa, R. (2014). Análisis estadístico con el SPSS. Bolivia: La Paz. Recuperado en: http://www.estadisticacondago.com/algebra\%20lineal/MANUAL\%20SPSS\%202014.pdf (Junio 1 de 2018) 